\title{
Drug repositioning for orphan genetic diseases through Conserved Anticoexpressed Gene Clusters (CAGCs)
}

\author{
Ivan Molineris, Ugo Ala, Paolo Provero* and Ferdinando Di Cunto*
}

\begin{abstract}
Background: The development of new therapies for orphan genetic diseases represents an extremely important medical and social challenge. Drug repositioning, i.e. finding new indications for approved drugs, could be one of the most cost- and time-effective strategies to cope with this problem, at least in a subset of cases. Therefore, many computational approaches based on the analysis of high throughput gene expression data have so far been proposed to reposition available drugs. However, most of these methods require gene expression profiles directly relevant to the pathologic conditions under study, such as those obtained from patient cells and/or from suitable experimental models. In this work we have developed a new approach for drug repositioning, based on identifying known drug targets showing conserved anti-correlated expression profiles with human disease genes, which is completely independent from the availability of 'ad hoc' gene expression data-sets.

Results: By analyzing available data, we provide evidence that the genes displaying conserved anti-correlation with drug targets are antagonistically modulated in their expression by treatment with the relevant drugs. We then identified clusters of genes associated to similar phenotypes and showing conserved anticorrelation with drug targets. On this basis, we generated a list of potential candidate drug-disease associations. Importantly, we show that some of the proposed associations are already supported by independent experimental evidence.

Conclusions: Our results support the hypothesis that the identification of gene clusters showing conserved anticorrelation with drug targets can be an effective method for drug repositioning and provide a wide list of new potential drug-disease associations for experimental validation.
\end{abstract}

\section{Background}

Orphan diseases (OD) are commonly defined as rare disorders with prevalence of less than 200000 cases in the US or of less than 1:2000 in Europe. Despite the low frequency of the single disorders, OD represent an extremely important medical and social challenge, because the $\sim 6000$ known rare diseases affect about $10 \%$ of the population in developed countries and because only in less than $5 \%$ of OD a treatment is available [1]. The search of possible targets and strategies for OD therapy is very actively pursued by basic science. However, translating the resulting knowledge into new drugs is complicated by the high costs of pre-clinical and clinical research, in comparison with the small potential for

\footnotetext{
*Correspondence: paolo.provero@unito.it; Ferdinando.dicunto@unito.it Molecular Biotechnology Centre, Department of Molecular Biotechnology

and Health Sciences, University of Torino, Via Nizza 52, 10126, Torino, Italy
}

revenue to pharmaceutical industry, because of the small market size [1]. Drug repositioning, i.e. finding new indications for approved drugs, represents to date one of the most cost- and time-effective strategies to identify a therapy for at least a subset of OD [2]. The rational basis for drug repositioning is given by the complex relationships between disease phenotypes, their underlying molecular mechanisms and the drugs that target them [3,4]. Indeed, it is increasingly recognized that the instances in which a clinical syndrome can be associated to a single molecular mechanism, which can be targeted by a very specific drug, is the exception rather than the rule in clinical practice. In particular, on one hand rare diseases and common disorders very often share some of their clinical features and underlying pathogenetic mechanisms $[3,4]$. On the other hand, the pathogenetic mechanisms of rare and common disorders often impinge on

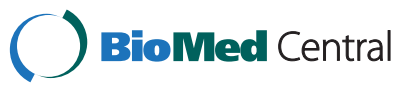


the function of entire molecular networks or functional modules, rather than on the function of single genes $[3,4]$. Finally, most of the chemical compounds approved for therapy are capable to bind and modify multiple molecular entities, producing a complex mixture of ontarget and off-target effects [5]. On the basis of this complexity, it is not surprising that in many cases a pharmacological compound originally approved for a common disorder has turned out to be useful to treat phenotypically related, or even apparently unrelated rare diseases, and vice versa $[2,6,7]$. So far, these discoveries have mostly been achieved serendipitously through the clinical monitoring of drug effects. However, in recent years, the availability of huge information on the genetic basis of human disorders, on gene regulation, on protein structure and on drug-target interactions has generated unprecedented opportunities to pursue drug repositioning on a more rational ground [8]. Accordingly, several computational approaches have been developed to discover unrecognized or non-explicit connections between drugs, targets and diseases. Many of these strategies have used structural similarity between ligands and target proteins, together with literature mining and protein-protein interaction maps, to produce wide drugtarget networks, allowing to infer new potential drugtarget associations [8-10]. Genome wide association studies have been used with the same purpose [11]. Gene expression data represent a very rich alternative resource for inferring non-obvious relationships between drugs and drug target genes, which can potentially be used for drug repositioning [12-14]. This approach is based on the commonly accepted principle that genes implicated in the same functional modules tend to display very similar expression patterns under physiological conditions and after internal or external perturbations. The strongest initiative in this sense is the Connectivity Map (CMap), a collection of genome-wide transcriptional expression data from cultured human cells treated with bioactive small molecules that allows the discovery of functional connections between drugs, genes and diseases through the analysis of common gene-expression changes [15,16]. A network-based analysis of this resource has allowed to predict similarities in drug effect and mechanism of action, and to "reposition" a Rhokinase inhibitor as an enhancer of cellular autophagy $[13,14]$. Another interesting example was the identification of ARA-C as a possible drug for Ewing sarcoma, based on the finding that the administration of this molecule to tumor cells negatively modulates a EWS/FLI oncoprotein-dependent gene signature [17]. Nevertheless, the potential of gene coexpression for proposing new associations between diseases, drug targets and drugs is still largely unexplored. The main limitation of the current approaches is that they mostly require gene expression profiles directly relevant to the condition under study, such as those obtained from patient cells and/or from suitable experimental models $[13,16,17]$. Unfortunately, these resources are not available in the majority of rare diseases. In this work we have developed a new computational strategy that may in part overcome this limitation. Our approach is based uniquely on the search for conserved anti-correlation between known drug targets and human disease genes, performed on public microarray databases. On this basis, we propose new potential candidate drug targets and drugs for rare human diseases for which no specific gene expression data are available.

\section{Results and discussion}

\section{Generation and characterization of Conserved Anticorrelated Gene Clusters (CAGC)}

Dissection of the molecular basis of many diseases has revealed that abnormal phenotypes are caused in most cases by the derangement of entire functional modules, due to single gene defects, to a combination of genetic abnormalities or to the interaction between gene variants and environmental factors [18,19]. The correct organization of these modules depends on a delicate equilibrium between positive and negative regulatory interactions. Positive functional interactions within a functional module are very often reflected by a highly similar expression profile (coexpression) of the genes that compose it. Indeed, it has been widely shown, through the systematic analysis of gene coexpression, that genes displaying very similar expression profiles tend to be functionally correlated [20] and that mutations affecting coexpressed genes tend to produce similar clinical syndromes [21-23]. Importantly, it has also been shown that these correlations are much stronger for genes that are consistently coexpressed across different species (conserved coexpression) [21,23-25]. Negative correlations have been much less investigated under this perspective. However, it has been suggested that if two genes display strongly anti-correlated expression profiles they may act in opposing functional modules or they may play opposing roles within the same functional module [26-28]. Potentially, this scenario is of great pharmacological relevance, because it would imply that the overall output of some disease-relevant functional modules could be positively or negatively modified by modulating the function of anti-correlated genes (Figure 1). Therefore, we decided to systematically analyze whether groups of mammalian genes displaying consistent anti-correlation with a specific gene are functionally characterized and are associated to similar disease phenotypes. To this aim, we studied a large, manually annotated microarray dataset downloaded from the Gene Expression Omnibus [29], covering many tissues, cell types and experimental 


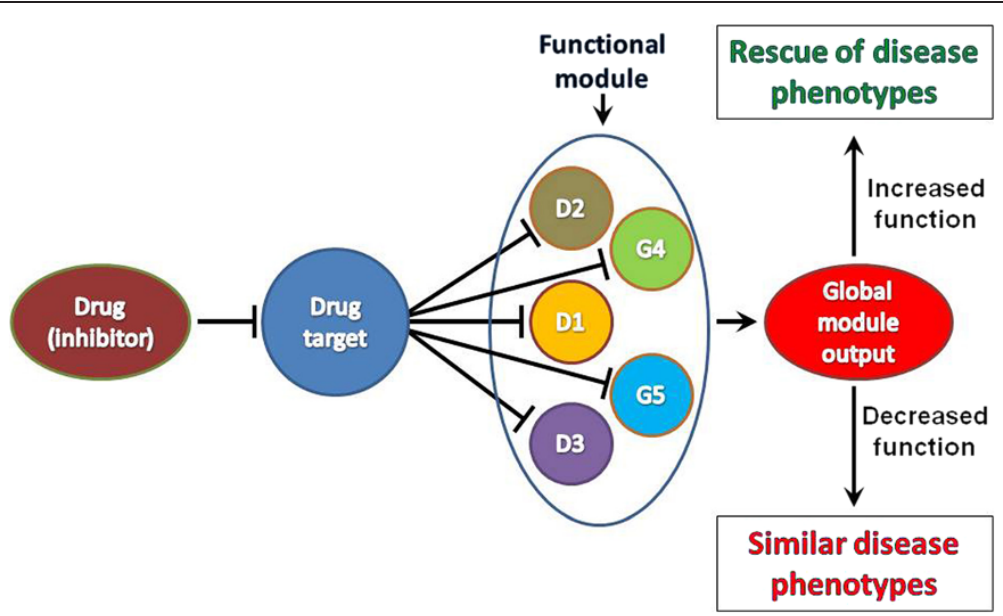

Figure 1 Schematic representation of how the antagonism between a drug target and a functional module containing functionally correlated genes, which may be implicated (D) or may be not implicated (G) in similar disease phenotypes, could be exploited to modify the outcome of genetic mutations.

conditions in both human (5188 experiments) and mouse (2310 experiments) [30]. We built generic and tissue-specific CAGC using a ranking-based procedure, very similar to the one that we previously used to identify conserved coexpression clusters $([21,30]$, see materials and methods for the details). In synthesis, the CACG for any particular gene of interest $(g)$, referred to as the centre, is composed of the genes that fall in the bottom 1\% correlation rank of $g$ in both human and mouse (a cluster does not includes the centre itself). We then compared the Gene Ontology (GO) functional annotation [31] of the obtained clusters with the annotation of random clusters of same size. Genes composing CAGCs were strongly enriched for genes annotated to the same GO keywords (Figure 2A). This was an expected result $[20,25,30]$, since genes composing a CAGC display strongly correlated expression profiles (data not shown). Interestingly, for clusters that showed at least one GO keyword enriched with a Bonferroni corrected p-value of 0.05 or less, $44 \%$ of the centre genes were annotated to at least one of the significant keywords. In addition, and most importantly, we found that the CAGCs are significantly enriched for genes associated to genetic diseases characterized by similar
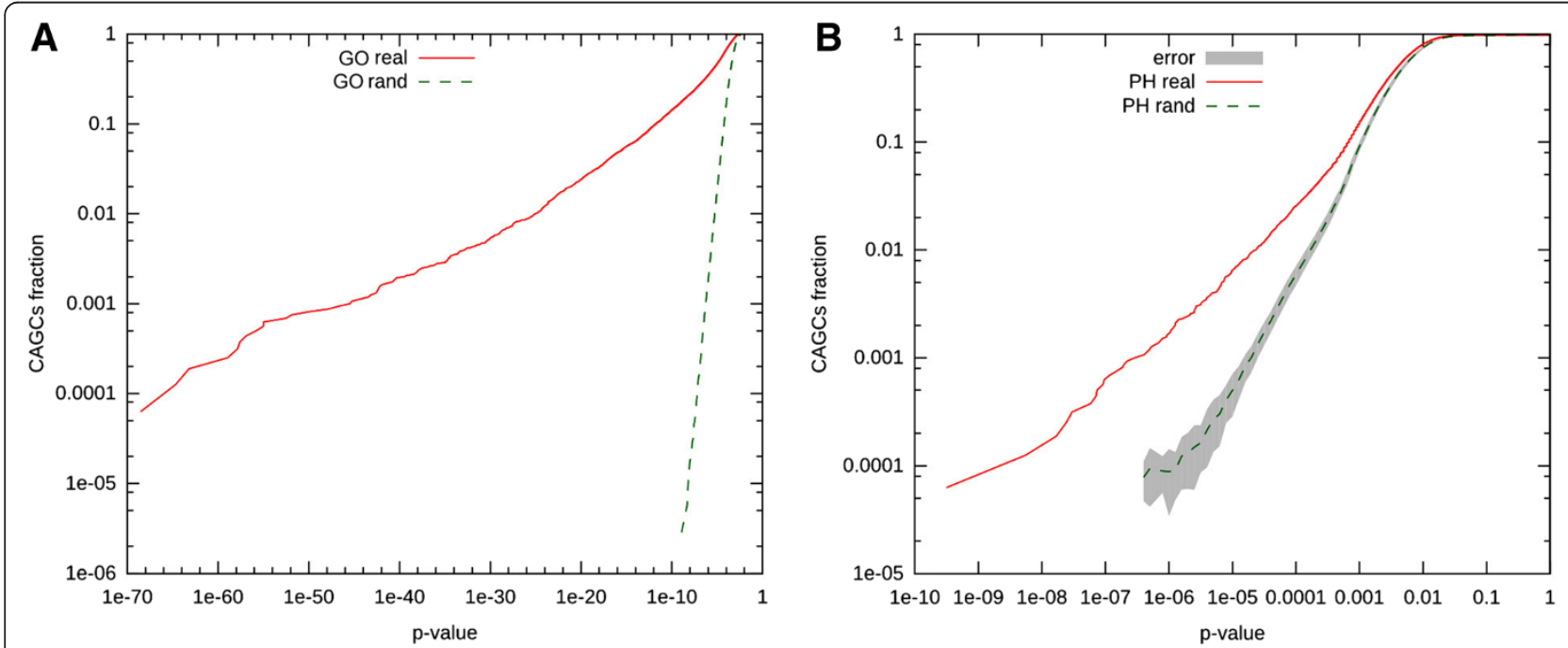

Figure 2 Cumulative distribution of functional index for GO categories and OMIM phenotypes. Cumulative log-log distribution of functional index (see methods) for A) GO categories, B) OMIM phenotypes (PH). Continuous curves represent the real data, while dashed curves result from 100 random permutation of real data (rand). Grey band in B corresponds to the standard deviation of the 100 randomly permuted datasets. The same band is not reported for panel A, since its thickness is comparable to the line thickness. In both cases the difference of real and permuted data is highly significant (P-value $<10 \mathrm{e}-256$ Kolmogorov-Smirnov test). 
phenotypes (Figure 2B), as defined by MimMiner [32]. However, when in these cases the centre genes were also associated to mendelian disorders, we found that the MimMiner scores calculated between the disease associated to the centre gene and those associated to the other genes of CAGC never reached the 0.4 similarity threshold. Altogether, these results indicate that, although the genes that compose a CAGC and the centre of the same clusters frequently work in the same functional modules, the phenotypic consequences of a mutation of the centre gene are different from the consequences of a mutation of the genes composing the corresponding CAGC. This scenario is compatible with the idea that the genes of a CAGC and its centre gene may play functionally antagonistic roles.

\section{GAGCs centered on drug targets are significantly modulated by treatment with the relevant drug}

We then asked whether pharmacological modulation of CAGCs centres may actually lead to a modulation of the anticorrelated genes. To this aim, we analyzed the gene expression data contained in CMap, which were obtained from cultured human cells treated with bioactive small molecules $[15,16]$. Moreover, we obtained the definition of genes as targets of specific drugs from analysis of the DrugBank database [33]. For every CAGC centered on a drug target associated to a molecule used in CMap (682 associations), we tested whether the genes in the CAGC are significantly upregulated or downregulated upon drug treatment (Mann-Whitney U test). We performed 8435 tests, 3510 of which were significant with a False Discovery Rate $($ FDR $)<0.05$. Importantly in 3428 cases the median ranks are consistent with an upregulation of the CAGC genes, while only in 82 cases it is consistent with a downregulation. The median rank distribution for all the CMap instances and the exemplar case of the genes composing the CAGC of ROCK1 (a target of Fasudil) in the CMap instance n. 436 (1e-05 M of Fasudil for $6 \mathrm{~h}$ in PC3 cells) are reported in Figure 3. Considering that the majority (76\%) of the drugs used in CMap and associated to a cluster significantly up- or down-regulated are classified as inhibitors in DrugBank, these results strongly validate the general idea that the genes anticorrelated with a drug target are upregulated when the function of the target gene is inhibited.

\section{Association of available drug targets to orphan genetic disorders through CAGCs}

On the basis of the above results, we thought to use CAGCs to associate know drug targets to mendelian genetic disorders. To this aim, we selected CAGCs characterized by two features: the centre of the CAGC is a drug target and the other genes that compose the cluster are significantly enriched for genes associated to related morbid map phenotypes [32] (Figure 4). Indeed, we would expect that the pharmacological downmodulation of some of these centres may compensate for the loss of at least some of the specific disease genes that are among them. Under this assumption, the drugs that are capable of inhibiting the CAGC centre would become potential candidates for all the related
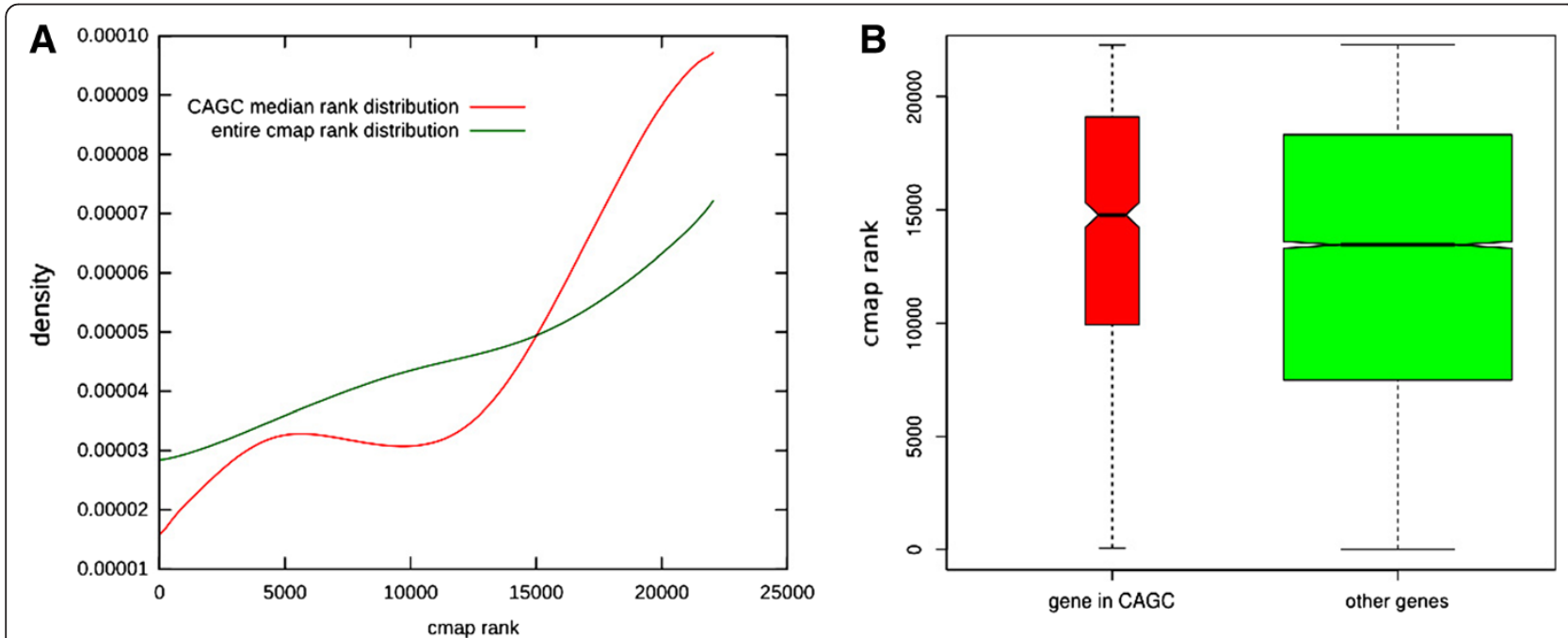

Figure 3 Cmap rank in CAGC genes. Panel A shows the gene rank distribution for all genes and all cmap instances (green curve) and the distribution of the median gene rank of each CAGC whose center is a known drug target (red curve). Note that the gene rank is defined as the highest rank among the probesets associated to the gene, which explains the non-uniform distribution of all genes. As an exemplar case, in panel $\mathbf{B}$ we show a box-plot comparing, for the cmap instance n. 436 (1e-05 M of Fasudil for $6 \mathrm{~h}$ in PC3 cells), the genes in the CAGC of ROCK1 (a known target of Fasudil) with all the other genes. The rank of the CAGC genes is significantly higher (Pvalue $<3.4 \mathrm{e}-05 \mathrm{Mann}-\mathrm{Whitney} \mathrm{U}$ test), implying that the inhibition of the CAGC center by the drug leads to a general overexpression of the genes that compose its CAGC. 


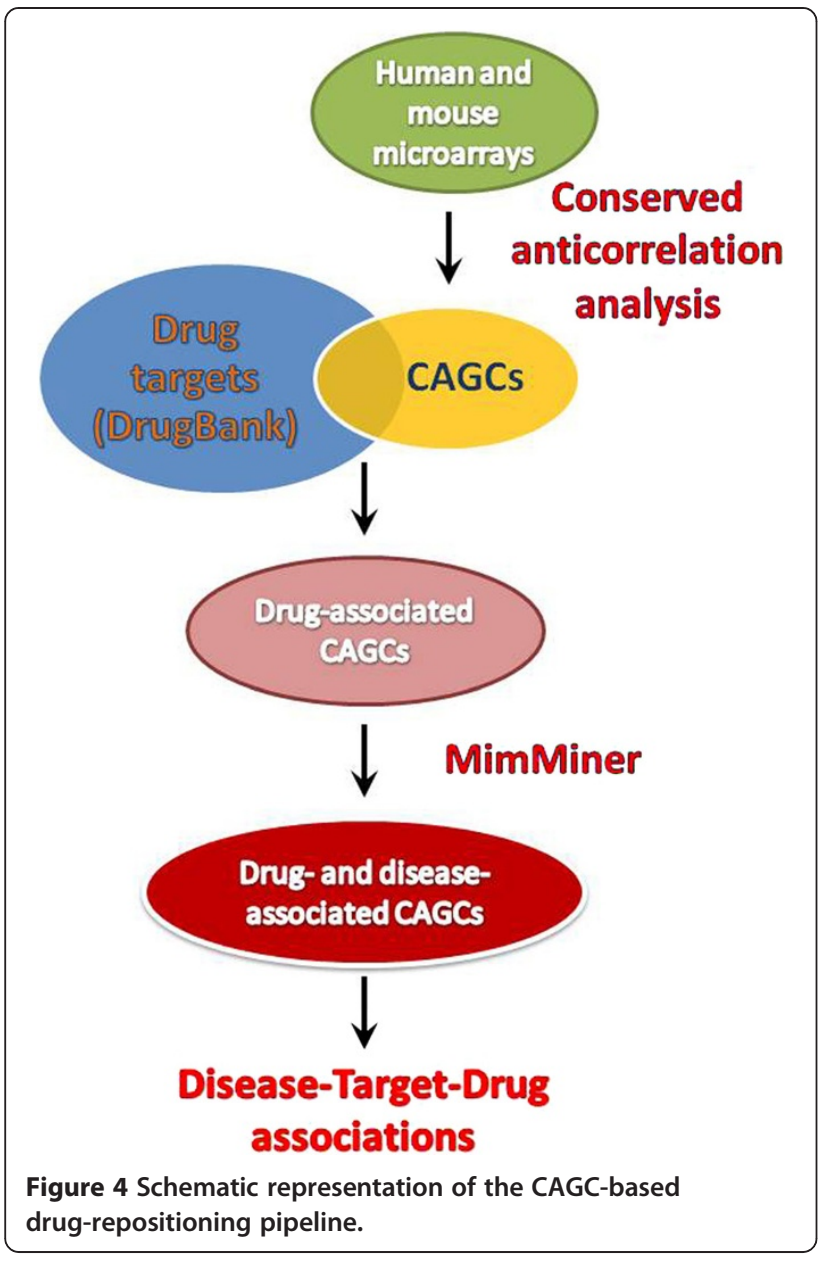

mendelian disorders associated to the cluster. In other words, we propose a drug as possible treatment for a genetic disease when the responsible gene (DG) belongs to an anticorrelation cluster that has the drug target as the centre and that is significantly enriched for other genes implicated in disorders similar to the disorder resulting from DG mutation. Using this strategy, we obtained the list of drug targets/disease associations reported in the Additional file 1. The list could contain both completely novel associations or associations which are already supported by previous evidence. Although we would expect most of the associations to be novel, finding a significant number of associations supported by literature could represent an important validation of our method. In consideration of the difficulty of automatically assessing this point, we decided to systematically inspect the list by expert analysis. The most prominent results are reported below.

- Calmodulins and malformative genetic syndromes Calmodulins 1 and 3 (CALM1 and CALM3) are, potentially, the most prominent targets underscored by our approach. Indeed, they are represented in
426 of the associations reported in the Additional file 1 , on a total of 2973. Since the two genes are strongly coexpressed (data not shown), the corresponding CAGCs were largely overlapping. Interestingly, these CACGs were strongly enriched for genes implicated in very complex and heterogeneous genetic syndromes. However, although many of these disorders are characterized by abnormalities of skeletal development, considering the high number of involved genes, the crucial role of calcium/calmodulin in many different cellular processes and the high number of drugs associated in DrugBank to Calmodulins, we consider this as a likely non-specific result of our analysis, which underscores the potential pitfalls of our method.

- Drug targets for epileptic syndromes Epileptic seizures represent a frequent neurological condition, affecting approximately $3 \%$ of the population and represent one of the most common symptoms of genetic disorders affecting the Central Nervous System (CNS). Accordingly, many diseases represented in our list are characterized by epileptic seizures (Additional file 1). Interestingly, most of these disorders have been linked by our pipeline to at least two strongly validated drug targets: histone deacetylase 1 (HDAC1) and translocator protein $(18 \mathrm{kDa})$ (TSPO). HDAC1 is one of the targets of valproic acid, one of the most prominent anticonvulsant agents currently used in chronic epilepsy management [34]. TSPO, a conserved mitochondrial protein implicated in cholesterol transport and biosynthesis, is one of the targets of some benzodiazepines, which represent another prominent pharmacological class in the management of seizures $[35,36]$. In particular, although the most prominent targets of benzodiazepines are GABA receptors, it has been proposed that TSPO-binding compounds may contribute to anticonvulsant effects by regulating the synthesis of neurosteroids [35]. Another interesting gene highlighted by our analysis for the same syndromes was the prolyl-4-isomerase beta (P4HB). Indeed, although this case is by far less validated, if compared to the previous two proteins, P4HB has recently been proposed as a target for new antiepileptogenic drug design [37].

- Drug targets for autism spectrum disorders Besides to epileptic syndromes, HDAC1 was identified as possible target also for the autismrelated disorder AUTS10 (MIM: 611016). We consider this result at least partially validated by the recent finding that $\mathrm{HDAC} 1$ inhibitors ameliorate social cognition and cell adhesion molecule plasticity deficits in a rodent model of autism spectrum 
disorder [38]. Similarly, we consider the association between prostaglandin-endoperoxide synthase 1 (PTGS1) and AUTSX2 partially validated by the finding that significantly elevated levels of prostaglandin E2 (PGE2) have been found in autistic patients [39].

- Drug targets for heart failure syndromes and for neuromuscular disorders

The Ser/Thr kinase ROCK1, a downstream effector of the cytoskeletal modulator RhoA small GTPase [40], was associated by our pipeline to 57 different genetic disorders, characterized by a high prevalence of muscular, cardiac and neurodegenerative phenotypes (Table 1).

Interestingly enough, specific inhibitors of this protein, such as the FDA approved Fasudil, have displayed beneficial effects in experimental animal models of some diseases included in the list. Indeed, ROCK1 has been shown to play an important role in the transition from cardiac hypertrophy to failure in mice [41] and the administration of ROCK1 inhibitors has repeatedly been associated to beneficial effects in non genetic models of heart failure [42-45]. Even more strikingly, it has been recently shown that Fasudil improves survival and promotes skeletal muscle development in a mouse genetic model of spinal muscular atrophy [46], one of the prominent diseases included in our list. Moreover, it is interesting to notice that ROCK1 expression has been correlated to disease progression in an animal model of ALS [47], although in these case a beneficial use of ROCK inhibitors has not been so far reported. A beneficial activity of ROCK inhibitors has also been documented in experimental models of myopathies. In particular, in muscle of dystrophin/utrophin double-knockout, which represent an intensely studied model of Duchenne muscular dystrophy, the RhoA/ROCK1 pathway is hyperactive, leading to a reduction of the myogenic potential of muscle stem cells [48]. Accordingly, treatment with a ROCK1 inhibitor improves the myogenic potential of stem cells and increases muscle regeneration in vivo [48]. A second interesting target for muscular disorders is given by the IKBKB (IKK- $\beta$ ) protein, a Ser/Thr kinase that phosphorylates and inactivates inhibitors of the NF-kB pathway [49] and which we found as potential target for four different myopathies. Indeed, not only it is well known that the NF-kB signalling is abnormally activated in myopathies such as Duchenne muscular dystrophy [50] and in limb-girdle muscular dystrophy [51], but it has been proved that NF-kB activation may functionally contribute to muscle degeneration [52]. Accordingly, it has been recently shown that NF-kB inhibition may improve muscle pathology and regeneration in the Mdx mouse model $[53,54]$.

\section{Conclusions}

We have provided proof of principle that the analysis of anti-correlated gene expression profiles could be systematically used as an innovative approach to explore the complex space of the interactions between genes, drugs and disease phenotypes. Moreover, we have implemented a predictive strategy capable to propose new associations of available drug targets and drugs to orphan genetic disorders. We have applied this approach to produce a list of such associations, which we provide as an open source of experimentally testable predictions, to be validated in cellular or animal disease models. On the basis of the validated or partially validated examples, it would seem to us reasonable to anticipate that, at least in some of the proposed cases, the new associations will turn out to be relevant not only to explore the possible use of the proposed drug targets in such disorders, but also to address new possible molecular mechanisms leading from gene inactivation to disease phenotypes. As a final remark, although we have only shown how this principle can be applied to the problem of drug repositioning, we can envisage that similar approaches could be used to help addressing other outstanding problems of drug development, such as predicting the possible side effects of inhibiting or stimulating new drug targets.

\section{Methods}

Definition and evaluation of CAGC

We studied a large, manually annotated, microarray dataset downloaded from the Gene Expression Omnibus (GEO), based on Affymetrix Plus 2 platform, covering many tissues, cell types and experimental conditions in both human (5188 experiments) and mouse (2310 experiments) [30]. Following procedures and considerations similar to those previously described in [21] we built tissue-specific conserved anti-coexpression networks. We first generated single species anti-coexpression gene networks (SAN) and then integrated them on the basis of human-mouse orthology. SANs were generated by first calculating the Pearson correlation coefficients of every microarray probeset with all the other probesets. A directed edge was established from probeset $\mathrm{p} 1$ to probeset $\mathrm{p} 2$ if $\mathrm{p} 1$ fell within the top $1 \%$ probesets in terms of anti-correlation with $\mathrm{p} 2$. These directed networks were then converted into undirected SANs by requiring a reciprocal $<1 \%$ ranking. We mapped each probeset to corresponding Entrez Gene identifiers using the Affymetrix na26 annotation, then an undirected edge was established between two Entrez gene G1 and G2 if there was at least one edge from a probeset assigned to 
Table 1 List of the genetic disorders associated to the ROCK1 target

\begin{tabular}{|c|c|c|}
\hline OMIM ID & Disease name & P-value \\
\hline 611126 & Deficiency of a Acyl-coa dehydrogenase family, member 9. & $1.5 \mathrm{e}-05$ \\
\hline 201450 & Deficiency of acyl-coa dehydrogenase, medium-chain. & $4.4 \mathrm{e}-05$ \\
\hline 201470 & Deficiency of acyl-coa dehydrogenase, short-chain. & $2.2 \mathrm{e}-05$ \\
\hline 201475 & Deficiency of acyl-coa dehydrogenase, very long-chain. & $4.2 \mathrm{e}-08$ \\
\hline 608810 & Alpha-b crystallinopathy. & $1.5 e-05$ \\
\hline 205200 & Amyotrophic lateral sclerosis, juvenile, with dementia. & $5.1 \mathrm{e}-05$ \\
\hline 600996 & Arrhythmogenic right ventricular dysplasia, familial, 2; arvd2. & $7.9 \mathrm{e}-05$ \\
\hline 609160 & Arrhythmogenic right ventricular dysplasia, familial, 7. & $1.3 e-05$ \\
\hline 604377 & Aardioencephalomyopathy, fatal infantile, due to cytochrome c oxidase. & 4.7e-09 \\
\hline 602067 & Cardiomyopathy, dilated, 1f; cmd1f. & $5.9 \mathrm{e}-05$ \\
\hline 192600 & Cardiomyopathy, familial hypertrophic 1; cmh1. & $7.4 \mathrm{e}-05$ \\
\hline 115210 & Cardiomyopathy, familial restrictive, $1 ;$ rcm 1 . & $7.1 \mathrm{e}-05$ \\
\hline 212350 & Cataract and cardiomyopathy. & $4.6 \mathrm{e}-07$ \\
\hline 601253 & Caveolin 3; cav3. & $7.8 \mathrm{e}-05$ \\
\hline 609060 & Combined oxidative phosphorylation deficiency 1 ; coxpd1. & $6.3 e-05$ \\
\hline 610505 & Combined oxidative phosphorylation deficiency 3; coxpd3. & $4.4 \mathrm{e}-08$ \\
\hline 611719 & Combined oxidative phosphorylation deficiency 5 ; coxpd5. & $9.9 \mathrm{e}-07$ \\
\hline 300257 & Danon disease. & $6.1 e-06$ \\
\hline 602668 & Dystrophia myotonica 2; dm2. & $3.6 \mathrm{e}-05$ \\
\hline 158900 & Facioscapulohumeral muscular dystrophy 1a; fshmd1a. & $2.4 \mathrm{e}-05$ \\
\hline 229300 & Friedreich ataxia $1 ;$ frda. & $5.5 e-05$ \\
\hline 253800 & Fukuyama congenital muscular dystrophy; fcmd. & $8.8 \mathrm{e}-05$ \\
\hline 232300 & Glycogen storage disease ii. & $4.9 \mathrm{e}-06$ \\
\hline 232400 & Glycogen storage disease iii. & $5.3 e-06$ \\
\hline 261740 & Glycogen storage disease of heart, lethal congenital. & $5.7 e-06$ \\
\hline 261670 & Glycogen storage disease $x ;$ gsd10. & $4.4 \mathrm{e}-06$ \\
\hline 600737 & Inclusion body myopathy 2, autosomal recessive; ibm2. & $8.8 \mathrm{e}-05$ \\
\hline 167320 & Inclusion body myopathy with early-onset paget disease and frontotemporal. & $5.3 e-05$ \\
\hline 147421 & Inclusion body miositis. & $2.4 \mathrm{e}-05$ \\
\hline 606183 & Laryngeal abductor paralysis with cerebellar ataxia and motor neuropathy. & $1.9 \mathrm{e}-05$ \\
\hline 220111 & Leigh syndrome, French-Canadian type; Isfc. & $7.8 \mathrm{e}-05$ \\
\hline 300322 & Lesch-nyhan syndrome; Ins. & $8.9 e-05$ \\
\hline 109150 & Machado-joseph disease; mjd. & $8.1 \mathrm{e}-05$ \\
\hline 248800 & Marinesco-sjogren syndrome; mss. & $7.9 e-05$ \\
\hline 252011 & Mitochondrial complex ii deficiency. & $6.7 e-06$ \\
\hline 609560 & Mitochondrial DNA depletion syndrome, myopathic form. & $3.4 \mathrm{e}-08$ \\
\hline 600462 & Mitochondrial myopathy and sideroblastic anemia; mlasa. & $7.2 \mathrm{e}-05$ \\
\hline 500002 & Mitochondrial myopathy with diabetes. & $3.1 \mathrm{e}-06$ \\
\hline 251950 & Mitochondrial myopathy with lactic acidosis. & $2.5 e-05$ \\
\hline 610773 & Mitochondrial phosphate carrier deficiency. & $5.5 \mathrm{e}-08$ \\
\hline 310200 & Muscular dystrophy, duchenne type; dmd. & $9.5 \mathrm{e}-05$ \\
\hline 605809 & Myasthenia, familial infantile, 1. & $2.3 e-07$ \\
\hline 610542 & Myasthenia, limb-girdle, with tubular aggregates. & $4.9 \mathrm{e}-06$ \\
\hline 254210 & Myasthenic syndrome, congenital, associated with episodic apnea. & $5.2 \mathrm{e}-07$ \\
\hline
\end{tabular}




\section{Table 1 List of the genetic disorders associated to the ROCK1 target (Continued)}

\begin{tabular}{|c|c|c|}
\hline 255125 & Myopathy with lactic acidosis, hereditary; hml & $3.9 \mathrm{e}-07$ \\
\hline 609500 & Myopathy, autophagic vacuolar, infantile-onset. & $3.6 \mathrm{e}-05$ \\
\hline 609200 & Myotilinopathy. & $2.6 \mathrm{e}-05$ \\
\hline 258450 & Progressive external ophthalmoplegia with mitochondrial dna deletions. & $1.2 \mathrm{e}-05$ \\
\hline 609286 & Progressive external ophthalmoplegia with mitochondrial dna deletions. & $2.1 \mathrm{e}-06$ \\
\hline 212138 & Solute carrier family 25 (carnitine/acylcarnitine translocase), member. & $4.3 e-06$ \\
\hline 103220 & Solute carrier family 25 (mitochondrial carrier, adenine nucleotide. & $3.9 \mathrm{e}-06$ \\
\hline 604360 & Spastic paraplegia 11, autosomal recessive; spg11. & $9.4 \mathrm{e}-05$ \\
\hline 610250 & Spastic paraplegia 31, autosomal dominant; spg31. & $8.4 \mathrm{e}-05$ \\
\hline 183020 & Spinal muscular atrophy, segmental. & 7.7e-05 \\
\hline 253300 & Spinal muscular atrophy, type i; sma1. & $6.2 \mathrm{e}-05$ \\
\hline 222300 & Wolfram syndrome 1; wfs1. & $7.5 \mathrm{e}-05$ \\
\hline
\end{tabular}

P-value refers to the enrichment for phenotypically correlated genes detected in the corresponding CAGC.

G1 to a probeset assigned to G2 and vice versa (reciprocal $1 \%$ ranking). We then constructed conserved anticoexpression gene networks (CAGN) starting from SANs by mapping every Entrez Gene identifier to the corresponding Homologene cluster (build 63). Two human genes G1h and G2h were connected in the CAGN if 1) they were connected in the human SAN, 2) booth of them were associated one-to-one to mouse genes by Homologene and 3) the corresponding genes G1m and G2m were connected in the murine SAN.

We constructed 18 CAGNs: 15 are specific of a single tissue (Adipose tissue, Brain, Breast, Central Nervous System, Sensory ganglia, Gastrointestinal tract, Heart, Kidney, Liver, Lung, Lymphatic tissues, Ovary, Prostate, Skeletal muscle, Stratified Epithelium), meaning that both the human and the murine SAN contributing to the tissue specific CAGN are based on samples derived from the same tissue. The three remaining CAGNs are not tissue-specific, because one is based on all the samples contained in the database, the second is based on the samples deriving from all the normal human and mouse tissue while the third derives from tumor cells data. We have previously shown [30] that coexpression information deriving from various tissue-specific datasets and from heterogeneous non-tissue-specific datasets contains highly complementary information. We thus chose to merge all the 18 CAGNs into a single one that we used for subsequent analyses (the merged network contained 15954 genes). Finally, for each gene $\mathrm{G}$ present in the merged CAGN, we define a conserved anti-coexpression gene cluster (CAGC), composed by the nearest-neighbours $G^{\prime}$ of the gene $G$ in the merged CAGN. The gene G, the centre of the cluster, is not considered as a member of the cluster. CAGC are available as Additional file 2 on the journal web site and at the URL http://www.cbu.mbcunito.it/ts-coexp.

\section{Pheno-Clusters}

Genetic disease phenotypes described in OMIM were correlated on the basis of MimMiner [32]. MimMiner assigns a similarity score to all pairs of OMIM phenotype records, based on the text mining analysis of their phenotype descriptions. The pheno-cluster of the phenotype $\mathrm{P}$ is the set of genes directly associated by OMIM to P or to a phenotype $\mathrm{P}^{\prime}$ whose similarity with $\mathrm{P}$ is at least 0.4 . The reason for choosing this cutoff is that biologically meaningful relationships were mostly detected in phenotype pairs with a similarity score equal to or greater than this value [32].

\section{Drug-phenotype association}

We statistically associated drug targets to phenotypes by evaluating the overlap between the CAGC and PhenoClusters, through a Fisher exact test. We considered as significant the overlaps with $\mathrm{P}$ value $<1 \mathrm{e}-4$, corresponding to $2.5 \%$ Benjamini-Hochberg FDR.

We used as reference for known drugs and targets DrugBank version 3 [33]. If a drug targeted a CAGC centre and there was a statistically significant association between the CAGC and one or more phenotypes, we putatively inferred an effect of the drug for these phenotypes. The idea under this inference criterion is that, if a drug D inhibits its targets, D should increase the expression of the CAGC centered in $G$ with an effect on the associated phenotypes. If this inference criterion is valid, then we can predict that a drug will increase the expression of CAGCs centered in its targets. To validate this observation we used geneexpression profiles derived from the treatment of cultured human cells with a large number of perturbagens produced by the CMap project (build 2) [16]. For the purpose of the current validation CMap can be viewed as a matrix having genes on the rows and experiments on columns. Each experiment consists of a perturbation of a cell-line with a given concentration of a drug. 
The matrix values are properly rank-transformed ratios between normalized expression values of the gene in the treated cell-line and a control; the greater the value the greater the fold-change in expression of the gene in the case versus the control. CMap reports the data in terms of Affymetrix probesets, which we mapped to Entrez Gene using the standard Affymetrix annotation (version na32). Since many probesets can be associated to the same gene, in each CMap instance we define the gene rank as the rank associated to the probeset with the highest rank. This explains why the gene rank distribution tends to be growing (see Figure 3) while the distribution of probeset rank is uniform by construction. Moreover some ranks are lost since some probesets can't be associated to any gene. Since DrugBank and CMap use different drug identifiers, we were able to associate CMap data to only 682 DrugBank compound. We used Chembl (version 12) [55] as a third source of drug information to map drug identifiers. Given a CAGC associated to a drug $\mathrm{D}$, we expect that its genes are over-expressed in cell lines treated with the drug targeting the CAGC centre. We tested this hypothesis independently for each CMap experiment that uses the $\mathrm{D}$ (there are possibly many experiment using the same drug with different concentration or different cell-lines) with a two sided Mann-Whitney $U$ test. For each drug we performed $\mathrm{N} \times \mathrm{M}$ tests, where $\mathrm{N}$ is the number of CAGCs whose centre $G$ is targeted by $D$, and $M$ is the number of experiments in CMap that involve D.

\section{Functional enrichment statistical validation}

To verify that CAGC tend to contain genes sharing the same biological function we defined two functional indices, 1) for GO categories, 2) for pheno-clusters.

Given a CAGC we used the Fisher exact test to compute the p-value of the enrichment of genes in each GO category (pheno-cluster). The GO (pheno-cluster) functional index of a CAGC is then the p-value of the most significant GO category (pheno-clusters).

We computed the functional indices for each CAGC in the real network and in 100 random networks obtained by permuting node (gene) labels, and finally we compared the cumulative distribution of functional indices derived from real versus randomized networks using a KolmogorovSmirnov test (see Figure 2). GO data [31] were obtained from the NCBI Entrez Gene site. All the associations were considered, including those inferred from electronic annotation (IEA).

\section{Additional files}

Additional file 1: Table S1. List the associations between available drug targets and orphan genetic disorders obtained through CAGCs.

Additional file 2: Compressed zip version of tab-delimited text file, Full list of the Conserved Anti-coexpressed Gene Clusters.
Competing interests

The authors declare no competing interests.

\section{Authors' contributions}

IM participated in the design of the study, performed most the analysis and participated to manuscript writing. UA produced the anti-correlation networks. PP and FDC conceived, supervised the study, were responsible of the expert analysis of data reported in the Additional file 1 and wrote the manuscript. All authors read and approved the final manuscript.

\section{Acknowledgments}

This work was directly supported by Regione Piemonte (Project Acronym: Druidi: Drug Innovation and Discovery, to FDC) and indirectly by the Neuroscience Program of the "Compagnia di S.Paolo", by "Compagnia di San Paolo", Progetto d'Ateneo, Università di Torino 2011 to FDC. The funding of AIRC (Associazione Italiana Ricerca Cancro) to PP and of the Telethon

Foundation to FDC are also gratefully acknowledged.

Received: 15 April 2013 Accepted: 24 September 2013

Published: 2 October 2013

\section{References}

1. Denis A, Mergaert L, Fostier C, Cleemput I, Simoens S: A comparative study of European rare disease and orphan drug markets. Health Policy 2010, 97:173-9.

2. Sardana D, Zhu C, Zhang M, Gudivada RC, Yang L, Jegga AG: Drug repositioning for orphan diseases. Brief Bioinform 2011, 12:346-56.

3. Piro RM: Network medicine: linking disorders. Hum Genet 2012, 131:1811-20.

4. Chan SY, Loscalzo J: The emerging paradigm of network medicine in the study of human disease. Circ Res 2012, 111:359-74.

5. Guengerich FP: Mechanisms of drug toxicity and relevance to pharmaceutical development. Drug Metab Pharmacokinet 2011, 26:3-14.

6. da Fonseca MA, Casamassimo P: Old drugs, new uses. Pediatr Dent 2011, 33:67-74.

7. Padhy BM, Gupta YK: Drug repositioning: re-investigating existing drugs for new therapeutic indications. J Postgrad Med 2011, 57:153-60.

8. Ekins S, Williams AJ, Krasowski MD, Freundlich JS: In silico repositioning of approved drugs for rare and neglected diseases. Drug Discov Today 2011, 16:298-310.

9. Gottlieb A, Stein GY, Ruppin E, Sharan R: PREDICT: a method for inferring novel drug indications with application to personalized medicine. $\mathrm{Mol}$ Syst Biol 2011, 7:496.

10. Luo H, Chen J, Shi L, Mikailov M, Zhu H, Wang K, He L, Yang L: DRAR-CPI: a server for identifying drug repositioning potential and adverse drug reactions via the chemical-protein interactome. Nucleic Acids Res 2011 39:W492-8.

11. Sanseau P, Agarwal P, Barnes MR, Pastinen T, Richards JB, Cardon LR, Mooser $V$ : Use of genome-wide association studies for drug repositioning. Nat Biotechnol 2012, 30:317-20.

12. Clark PM, Dawany N, Dampier W, Byers SW, Pestell RG, Tozeren A: Bioinformatics analysis reveals transcriptome and microRNA signatures and drug repositioning targets for IBD and other autoimmune diseases. Inflamm Bowel Dis 2012, 18:2315-33.

13. Iorio F, Bosotti R, Scacheri E, Belcastro V, Mithbaokar P, Ferriero R, Murino L, Tagliaferri R, Brunetti-Pierri N, Isacchi A, et al: Discovery of drug mode of action and drug repositioning from transcriptional responses. Proc Natl Acad Sci USA 2010, 107:14621-6.

14. Iorio F, Isacchi A, di Bernardo D, Brunetti-Pierri N: Identification of small molecules enhancing autophagic function from drug network analysis. Autophagy 2010, 6:1204-5.

15. Lamb J: The Connectivity Map: a new tool for biomedical research. Nat Rev Cancer 2007, 7:54-60.

16. Lamb J, Crawford ED, Peck D, Modell JW, Blat IC, Wrobel MJ, Lerner J, Brunet JP, Subramanian A, Ross KN, et al: The Connectivity Map: using gene-expression signatures to connect small molecules, genes, and disease. Science 2006, 313:1929-35.

17. Stegmaier K, Wong JS, Ross KN, Chow KT, Peck D, Wright RD, Lessnick SL, Kung AL, Golub TR: Signature-based small molecule screening identifies cytosine arabinoside as an EWS/FLI modulator in Ewing sarcoma. PLoS Med 2007, 4:e122. 
18. Wang X, Dalkic E, Wu M, Chan C: Gene module level analysis: identification to networks and dynamics. Curr Opin Biotechnol 2008, 19:482-91.

19. Oti M, Brunner HG: The modular nature of genetic diseases. Clin Genet 2007, 71:1-11.

20. Eisen MB, Spellman PT, Brown PO, Botstein D: Cluster analysis and display of genome-wide expression patterns. Proc Natl Acad Sci USA 1998 95:14863-8.

21. Ala U, Piro RM, Grassi E, Damasco C, Silengo L, Oti M, Provero P, Di Cunto F: Prediction of human disease genes by human-mouse conserved coexpression analysis. PLoS Comput Biol 2008, 4:e1000043.

22. Miozzi L, Piro RM, Rosa F, Ala U, Silengo L, Di Cunto F, Provero P: Functional annotation and identification of candidate disease genes by computational analysis of normal tissue gene expression data. PLoS One 2008, 3:e2439.

23. Oti M, van Reeuwijk J, Huynen MA, Brunner HG: Conserved co-expression for candidate disease gene prioritization. BMC Bioinformatics 2008, 9:208.

24. Pellegrino M, Provero P, Silengo L, Di Cunto F: CLOE: identification of putative functional relationships among genes by comparison of expression profiles between two species. BMC Bioinformatics 2004, 5:179.

25. Stuart JM, Segal E, Koller D, Kim SK: A Gene-Coexpression Network for Global Discovery of Conserved Genetic Modules. Science 2003, 302:249-255.

26. Shatkay H, Edwards S, Wilbur WJ, Boguski M: Genes, themes and microarrays: using information retrieval for large-scale gene analysis. Proc Int Conf Intell Syst Mol Biol 2000, 8:317-28.

27. He F, Zhou Y, Zhang Z: Deciphering the Arabidopsis floral transition process by integrating a protein-protein interaction network and gene expression data. Plant Physiol 2010, 153:1492-505.

28. Dhillon IS, Marcotte EM, Roshan U: Diametrical clustering for identifying anti-correlated gene clusters. Bioinformatics 2003, 19:1612-9.

29. Barrett T, Suzek TO, Troup DB, Wilhite SE, Ngau WC, Ledoux P, Rudnev D, Lash AE, Fujibuchi W, Edgar R: NCBI GEO: mining millions of expression profiles-database and tools. Nucleic Acids Res 2005, 33:D562-6.

30. Piro RM, Ala U, Molineris I, Grassi E, Bracco C, Perego GP, Provero P, Di Cunto F: An atlas of tissue-specific conserved coexpression for functional annotation and disease gene prediction. Eur J Hum Genet 2011, 19:1173-80.

31. Ashburner M, Ball CA, Blake JA, Botstein D, Butler H, Cherry JM, Davis AP, Dolinski K, Dwight SS, Eppig JT, et al: Gene ontology: tool for the unification of biology. The Gene Ontology Consortium. Nat Genet 2000, 25:25-9.

32. van Driel MA, Bruggeman J, Vriend G, Brunner HG, Leunissen JA: A text-mining analysis of the human phenome. Eur J Hum Genet 2006, 14:535-42.

33. Knox C, Law V, Jewison T, Liu P, Ly S, Frolkis A, Pon A, Banco K, Mak C, Neveu V: DrugBank 3.0: a comprehensive resource for 'omics' research on drugs. Nucleic Acids Res 2011, 39:D1035-D1041.

34. Phiel CJ, Zhang F, Huang EY, Guenther MG, Lazar MA, Klein PS: Histone deacetylase is a direct target of valproic acid, a potent anticonvulsant, mood stabilizer, and teratogen. J Biol Chem 2001, 276:36734-41.

35. Dhir A, Rogawski MA: Role of neurosteroids in the anticonvulsant activity of midazolam. Br J Pharmacol 2012, 165:2684-91.

36. Lindemann P, Koch A, Degenhardt B, Hause G, Grimm B, Papadopoulos V: A novel Arabidopsis thaliana protein is a functional peripheral-type benzodiazepine receptor. Plant Cell Physiol 2004, 45:723-33.

37. Rowles J, Olsen M: Perspectives on the development of antioxidant antiepileptogenic agents. Mini Rev Med Chem 2012, 12:1015-27.

38. Foley AG, Gannon S, Rombach-Mullan N, Prendergast A, Barry C, Cassidy AW, Regan CM: Class I histone deacetylase inhibition ameliorates social cognition and cell adhesion molecule plasticity deficits in a rodent model of autism spectrum disorder. Neuropharmacology 2012, 63:750-60.

39. El-Ansary A, Al-Ayadhi L: Lipid mediators in plasma of autism spectrum disorders. Lipids Health Dis 2012, 11:160.

40. Fujisawa K, Fujita A, Ishizaki T, Saito Y, Narumiya S: Identification of the Rho-binding domain of p160ROCK, a Rho-associated coiled-coil containing protein kinase. J Biol Chem 1996, 271:23022-8.

41. Shi J, Zhang YW, Yang Y, Zhang L, Wei L: ROCK1 plays an essential role in the transition from cardiac hypertrophy to failure in mice. $J \mathrm{Mol} \mathrm{Cell}$ Cardiol 2010, 49:819-28.

42. Ichinomiya T, Cho S, Higashijima U, Matsumoto S, Maekawa T, Sumikawa K High-dose fasudil preserves postconditioning against myocardial infarction under hyperglycemia in rats: role of mitochondrial KATP channels. Cardiovasc Diabetol 2012, 11:28

43. Wang N, Guan P, Zhang JP, Chang YZ, Gu LJ, Hao FK, Shi ZH, Wang FY, Chu $L$ : Preventive effects of fasudil on adriamycin-induced cardiomyopathy: possible involvement of inhibition of RhoA/ROCK pathway. Food Chem Toxicol 2011, 49:2975-82

44. Guan SJ, Ma ZH, Wu YL, Zhang JP, Liang F, Weiss JW, Guo QY, Wang JY, Ji ES, Chu L: Long-term administration of fasudil improves cardiomyopathy in streptozotocin-induced diabetic rats. Food Chem Toxicol 2012, 50:1874-82.

45. Guo P, Wu C, Masaki T, Mori H, Nishiyama A: Subdose of fasudil suppresses myocardial fibrosis in aldosterone-salt-treated uninephrectomized rats. Pharmazie 2011, 66:716-9.

46. Bowerman M, Murray LM, Boyer JG, Anderson CL, Kothary R: Fasudil improves survival and promotes skeletal muscle development in a mouse model of spinal muscular atrophy. BMC Med 2012, 10:24.

47. Capitanio D, Vasso M, Ratti A, Grignaschi G, Volta M, Moriggi M, Daleno C, Bendotti C, Silani V, Gelfi C: Molecular signatures of amyotrophic lateral sclerosis disease progression in hind and forelimb muscles of an SOD1 (G93A) mouse model. Antioxid Redox Signal 2012, 17:1333-50.

48. Mu X, Usas A, Tang Y, Lu A, Wang B, Weiss K, Huard J: RhoA mediates defective stem cell function and heterotopic ossification in dystrophic muscle of mice. Faseb J 2013.

49. Mercurio F, Zhu H, Murray BW, Shevchenko A, Bennett BL, Li J, Young DB, Barbosa M, Mann M, Manning A, et al: IKK-1 and IKK-2: cytokine-activated IkappaB kinases essential for NF-kappaB activation. Science 1997, 278:860-6.

50. Monici MC, Aguennouz M, Mazzeo A, Messina C, Vita G: Activation of nuclear factor-kappaB in inflammatory myopathies and Duchenne muscular dystrophy. Neurology 2003, 60:993-7.

51. Baghdiguian S, Martin M, Richard I, Pons F, Astier C, Bourg N, Hay RT, Chemaly R, Halaby G, Loiselet J, et al: Calpain 3 deficiency is associated with myonuclear apoptosis and profound perturbation of the IkappaB alpha/NF-kappaB pathway in limb-girdle muscular dystrophy type $2 \mathrm{~A}$. Nat Med 1999, 5:503-11.

52. Acharyya S, Villalta SA, Bakkar N, Bupha-Intr T, Janssen PM, Carathers M, Li ZW, Beg AA, Ghosh S, Sahenk Z, et al: Interplay of IKK/NF-kappaB signaling in macrophages and myofibers promotes muscle degeneration in Duchenne muscular dystrophy. J Clin Invest 2007, 117:889-901.

53. Reay DP, Yang M, Watchko JF, Daood M, O'Day TL, Rehman KK, Guttridge DC, Robbins PD, Clemens PR: Systemic delivery of NEMO binding domain/ IKKgamma inhibitory peptide to young mdx mice improves dystrophic skeletal muscle histopathology. Neurobiol Dis 2011, 43:598-608.

54. Tang Y, Reay DP, Salay MN, Mi MY, Clemens PR, Guttridge DC, Robbins PD, Huard J, Wang B: Inhibition of the IKK/NF-kappaB pathway by AAV gene transfer improves muscle regeneration in older mdx mice. Gene Ther 2010, 17:1476-83.

55. Gaulton A, Bellis $\sqcup$, Bento AP, Chambers J, Davies M, Hersey A, Light Y, McGlinchey S, Michalovich D, Al-Lazikani B, et al: ChEMBL: a large-scale bioactivity database for drug discovery. Nucleic Acids Res 2012, 40:D1100-7.

doi:10.1186/1471-2105-14-288

Cite this article as: Molineris et al:: Drug repositioning for orphan genetic diseases through Conserved Anticoexpressed Gene Clusters (CAGCs). BMC Bioinformatics 2013 14:288.

\section{Submit your next manuscript to BioMed Central and take full advantage of:}

- Convenient online submission

- Thorough peer review

- No space constraints or color figure charges

- Immediate publication on acceptance

- Inclusion in PubMed, CAS, Scopus and Google Scholar

- Research which is freely available for redistribution 